\title{
Evaluation of Renal Fibrosis by Mapping Histology and Magnetic Resonance Imaging
}

\author{
Jiong Zhang ${ }^{\mathrm{a}}$ Yuanmeng $\mathrm{Yu}^{\mathrm{b}}$ Xiaoshuang Liu ${ }^{\mathrm{c}}$ Xiong Tang ${ }^{\mathrm{a}}$

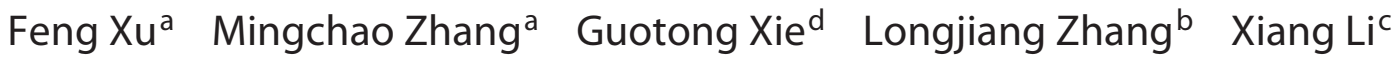 \\ Zhi-Hong Liu ${ }^{\mathrm{a}}$ \\ ${ }^{a}$ National Clinical Research Center of Kidney Diseases, Jinling Hospital, Second Military Medical University, Nanjing, \\ China; ${ }^{\circ}$ Department of Medical Imaging, Jinling Hospital, Nanjing University School of Medicine, Nanjing, China;

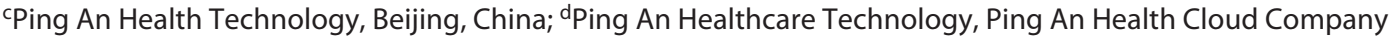 \\ Limited, Ping An International Smart City Technology Co., Ltd., Beijing, China
}

\section{Keywords \\ Chronic kidney disease - Peritubular capillaries . \\ Extracellular matrix · Diffusion - Weighted imaging · \\ Magnetic resonance elastography}

\begin{abstract}
Background: Renal fibrosis is a key driver of progression in chronic kidney disease (CKD). Recent advances in diagnostic imaging techniques have shown promising results for the noninvasive assessment of renal fibrosis. However, the specificity and accuracy of these techniques are controversial because they indirectly assess renal fibrosis. This limits fibrosis assessment by imaging in CKD for clinical practice. To validate magnetic resonance imaging (MRI) assessment for fibrosis, we derived representative models by mapping histology-proven renal fibrosis and imaging in CKD. Methods: Ninety-seven adult Chinese CKD participants with histology were studied. The kidney cortex interstitial extracellular matrix volume was calculated by the Aperio ScanScope system using Masson's trichrome slices. The kidney cortex microcirculation was quantitatively assessed by peritubular capillary density using CD34 staining. The imaging techniques included intravoxel incoherent motion diffusion-weighted imag-
\end{abstract}

karger@karger.com www.karger.com/kdd

Karger $\stackrel{\text { ' }}{5}$

GOPEN ACCESS
(C) 2021 The Author(s)

Published by S. Karger AG, Basel

This is an Open Access article licensed under the Creative Commons Attribution-NonCommercial-4.0 International License (CC BY-NC) (http://www.karger.com/Services/OpenAccessLicense), applicable to the online version of the article only. Usage and distribution for commercial purposes requires written permission. ing and magnetic resonance elastography (MRE) imaging. Relevant analyses were performed to evaluate the correlations between MRI parameters and histology variables. Multiple linear regression models were used to describe the relationships between a response variable and other variables. The best-fit lines, which minimize the sum of squared residuals of the multiple linear regression models, were generated. Results: MRE values were negatively associated with the interstitial extracellular matrix volume (Rho $=-0.397, p<$ 0.001 ). The best mapping model of extracellular matrix volume with the MRE value and estimated glomerular filtration rate (eGFR) we obtained was as follows: Interstitial extracellular matrix volume $=218.504-14.651 \times \operatorname{In}($ MRE $)-18.499 \times$ In(eGFR). DWI-fraction values were positively associated with peritubular capillary density $(R h o=0.472, p<0.001)$. The best mapping model of peritubular capillary density with DWI-fraction value and eGFR was as follows: Peritubular capillaries density $=17.914+9.403 \times(\mathrm{DWI}-$ fraction $)+0.112$ $\times$ (eGFR). Conclusions: The study provides histological evi-

Yuanmeng Yu: Current address: Department of MRI, The First People's Hospital of Yunnan Province, The Affiliated Hospital of Kunming University of Science and Technology, No. 157 Jinbi Road, Kunming 650032 , China. 
dence to support that MRI can effectively evaluate fibrosis in the kidney. These findings picture the graphs of the mapping model from imaging and eGFR into fibrosis, which has significant value for clinical implementation.

(C) 2021 The Author(s)

Published by S. Karger AG, Basel

\section{Introduction}

Chronic kidney disease (CKD) is an immense public health problem that affects approximately $10 \%$ of the world's population; it causes millions of deaths yearly and requires hundreds of thousands of people to use dialysis to stay alive $[1,2]$. Previously, known as kidney diseases, CKD is a condition characterized by a gradual loss of kidney function, which is recommended to be divided into 6 stages of worsening progression based on the GFR category and albuminuria category. Unfortunately, the level of estimated GFR does not always precisely reflect the extent of cortical interstitial fibrosis, which is the histological hallmark driver of the progression of kidney diseases $[3,4]$. The evaluation of interstitial fibrosis is therefore used to guide treatment and judge renal prognosis.

A number of previous magnetic resonance imaging (MRI) studies have demonstrated advances in the assessment of kidney diseases [5-11]. These advanced MRI techniques have been utilized to noninvasively assess renal fibrosis in diabetic nephropathy, CKD without diabetes, acute kidney injury [5], and allografts [8] indirectly. Magnetic resonance elastography (MRE) has shown success in quantitating the increased shear stiffness of liver tissue in the setting of hepatic fibrosis and cirrhosis [1214]. Earlier studies showed the feasibility of MRE in native and transplanted kidneys. However, recently, the results were variable from reports about advanced MRI techniques used for the noninvasive evaluation of fibrosis in kidney diseases. In their pilot study, Kirpalani et al. [8] showed the potential for MRE as a novel noninvasive measure of whole-allograft fibrosis burden, as it may predict future changes in kidney function. Their data suggested that whole-kidney stiffness correlated with the biopsy-derived Banff fibrosis score. Nevertheless, recently, at least 3 articles from native kidneys showed that MRE values had a negative relationship with fibrosis degree in IgA nephropathy [15], lupus nephritis [16], and diabetic nephropathy [17].

Moreover, diffusion-weighted MRI has been described as promising for the evaluation of renal fibrosis $[5,18$, 19]. In human kidneys, Inoue et al. [5] showed that DWIMRI was correlated with renal function and interstitial fibrosis in diabetic patients. Although correlation to renal fibrosis was observed, the additional role of perfusion and microcirculation in these associations was controversial [20].

Notably, there are multiple physical changes that occur in the kidney as it scars. First, accumulating evidence has demonstrated that progressive capillary loss occurs in parallel with kidney scarring, resulting in reduced blood flow and oxygen delivery to the tubular epithelial cells of the kidney parenchyma, which can drive further fibrogenesis. Thus, capillary loss and hypoperfusion are not only key features of the fibrosing kidney but also drivers of further fibrosis-associated injury $[18,21]$. A second important aspect was that, as the nephron scars, the deposited extracellular matrix volume increased, which may affect organ elasticity $[18,21]$. However, the gap between imaging, eGFR, and fibrotic lesions complicates the precise understanding and evaluation of CKD progression.

Therefore, the goal of this hypothesis-driven study was to validate MRI assessment for fibrosis by mapping histology-proven renal fibrosis and imaging in CKD. Two key fibrosis-associated histological features, peritubular capillary density and interstitial extracellular matrix volume, were investigated with intravoxel incoherent motion diffusion-weighted imaging (IVIM-DWI), MRE imaging, and renal function.

\section{Methods}

\section{Study Population}

We performed a cohort study of adult CKD patients whose data were retrieved consecutively from the Nanjing Glomerulonephritis Registry between January 2016 and December 2017. This research project was reviewed and approved by the Institutional Review Board of Jinling Hospital, Nanjing University School of Medicine, and conducted according to the principles of the Declaration of Helsinki. Informed written consent has been obtained from all participants. Patients were recruited and considered eligible if they underwent kidney biopsy and parallel IVIM-DWI and MRE examinations. The exclusion criteria for our study were as follows: (1) decline of consent; (2) claustrophobia or other standard MRI contraindications; and (3) patients with biopsy-proven acute kidney injury histology changes.

\section{Clinical and Laboratory Assessments}

Clinical and laboratory data were collected for MRI scanning and kidney biopsy. The eGFR was calculated using the CKD Epidemiology Collaboration (CKD-EPI) formula [22].

MRI Data Acquisition and Image Analyses

MR Data Acquisition

All MRI examinations were performed on a 3.0-T scanner (Discovery MR 750; GE Medical Systems, Milwaukee, WI, USA). 
All subjects in the current study had fasted at least $6 \mathrm{~h}$ before the scan. A 32-channel torso phased array coil was centered over the kidney. Patients were positioned feet first and supine. Axial multisection echo-planar DW MR imaging was performed. The relevant imaging parameters are summarized as we reported before [23]: 12-18 sections (section thickness, $6 \mathrm{~mm}$; intersection gap, $1 \mathrm{~mm}$ ); field of view, $38 \mathrm{~mm}$; matrix, $128 \times 128$; 6 signals acquired; and bandwidth, $250 \mathrm{~Hz}$ per pixel. The following 16 diffusion gradient $b$ values were used to acquire apparent diffusion coefficient (ADC) maps: 0, 10, 30, 50, 70, 100, 150, 200, 400, 800, 1,000, and $1,500 \mathrm{~s} / \mathrm{mm}^{2}$. The gradients were applied in 3 orthogonal directions and subsequently averaged to minimize the effects of diffusion anisotropy. A parallel imaging technique (modified sensitivity encoding) with a reduction factor of 2 was applied. Respiratory triggering was used with a minimum repetition time of $30 \mathrm{~ms}$ and a minimum echo time of $86.8 \mathrm{~ms}$ to reduce motion artifacts. Section positioning was identical to that used with the coronal T1weighted sequence and axial T2-weighted sequence. Mean acquisition time was 6 min.

The mechanical vibrations required for MRE were supplied by an active pneumatic driver system. The driver actuator was placed on the corresponding surface projection of the left kidney with ultrasound localization. MRE acquisitions were performed using a $60-\mathrm{Hz}$ vibration frequency and a breath-holding, flow-compensated two-dimensional echo-planar coronal MRE pulse sequence to derive magnitude images and a corresponding stiffness map. The imaging orientation was standardized to the coronal plane, with the frequency encoding direction superior to inferior. The MRE imaging parameters are shown in online suppl. Method 1 (see www. karger.com/doi/10.1159/000513332 for all online suppl. material).

Functional MR Imaging Evaluation

All original imaging data were independently analyzed and postprocessed with a GE workstation with a standard software package (Advantage Workstation Volume Share 5; GE Healthcare) by 2 well-experienced technicians who were blinded to the clinical and histological data. The IVIM-DWI and diffusion values were calculated on a pixel-by-pixel basis in 2 ways as our center reported before [23]. To separate the pure diffusion and pseudodiffusion contributions to signal decay, a biexponential diffusion model with a multistep approach was used. The region of interest (ROI) was manually placed as large as possible to include the kidney cortex in the upper, middle, and lower thirds of the kidney. Mean diffusion values were calculated for the cortex. Visible infarcts of renal parenchyma were identified and were excluded from the analysis. MRE and stiffness map analyses were performed as reported in the literature [8].

\section{Kidney Histology}

Ultrasound-guided kidney biopsies were performed within 3 days after MRI scanning. The kidney biopsy specimens were examined according to the standard procedures we performed before [24].

Aperio Quantification of Kidney Interstitial Extracellular Matrix Volume

Kidney interstitial fibrosis quantitation was evaluated as reported previously by our group [25]. Kidney sections stained with Masson's trichrome were scanned with an Aperio ScanScope system (Leica Biosystems, Germany) and analyzed using
ImageScope (Aperio). Kidney interstitial fibrosis was quantified according to the following formula: the percentage of the area of brilliant green staining/total section area in the cortex (except for the glomeruli).

\section{Measurement of Peritubular Capillaries Density}

CD34 (DAKO, Carpinteria, CA, USA) staining, which is specific for endothelial cells, was performed to label the peritubular capillaries [26]. The density of the peritubular capillaries was counted for each sample. The density was calculated by counting the total number of PTCs confined within each of 20 random 0.25 $\mathrm{mm}^{2}$ fields (each of these fields was delineated by a $1-\mathrm{cm}^{2}$ ocular grid that was viewed at $\times 400$ magnification), and the result was expressed as the average value per field [27].

\section{Statistical Analysis}

Continuous variables are expressed as the mean \pm standard deviation (25th and 75th percentiles), and categorical variables are expressed as frequency numbers. One-way ANOVA was used to compare normally distributed continuous data followed by the least significant difference test. Pearson or Spearman correlation was used to evaluate the correlations between the functional MRI parameters and histology variables, as appropriate. Categorical data were compared using the $\chi^{2}$ test. Statistical significance was defined as $p<0.05$.

\section{Multiple Linear Regression Models}

Multiple linear regression models were used to describe the relationships between a response variable and other variables. We used the $\operatorname{lm}$ () function of $R$ to create multiple linear regression models. The best-fit lines, which minimize the sum of squared residuals of the multiple linear regression models, were generated.

The equation for the multiple linear regression models is defined as follows:

$$
y=\beta 0+\beta 1 X 1+\beta 2 X 2,
$$

where $y$ is the response variable, $\mathrm{X}_{1}$ and $\mathrm{X}_{2}$ are the regressors, $\beta_{0}$ is an intercept, $\beta_{1}$ and $\beta_{2}$ are the regression coefficients, and $\varepsilon$ represents the error residuals. The least squares method is used to estimate $\beta_{0}, \beta_{1}$, and $\beta_{2}$. The model evaluation, $F$-statistic, and $p$ value sections have been given in online suppl. Method 2 .

\section{Results}

\section{Demographic and Clinicopathological Data}

A total of 97 eligible patients with CKD were studied (50 males, mean age $36.6 \pm 13.1$ years). Most of these patients had IgA nephropathy/Henoch Schönlein purpura nephritis $(n=38)$, minimal change disease/focal segmental glomerulosclerosis $(n=16)$, and diabetic nephropathy/obesity-related glomerulopathy $(n=14)$. In addition to primary glomerulonephritis patients, 11 lupus nephritis and 6 chronic interstitial nephritis patients were included. The demographic and CKD-related clinical data of these patients are shown in online suppl. Table 1. 


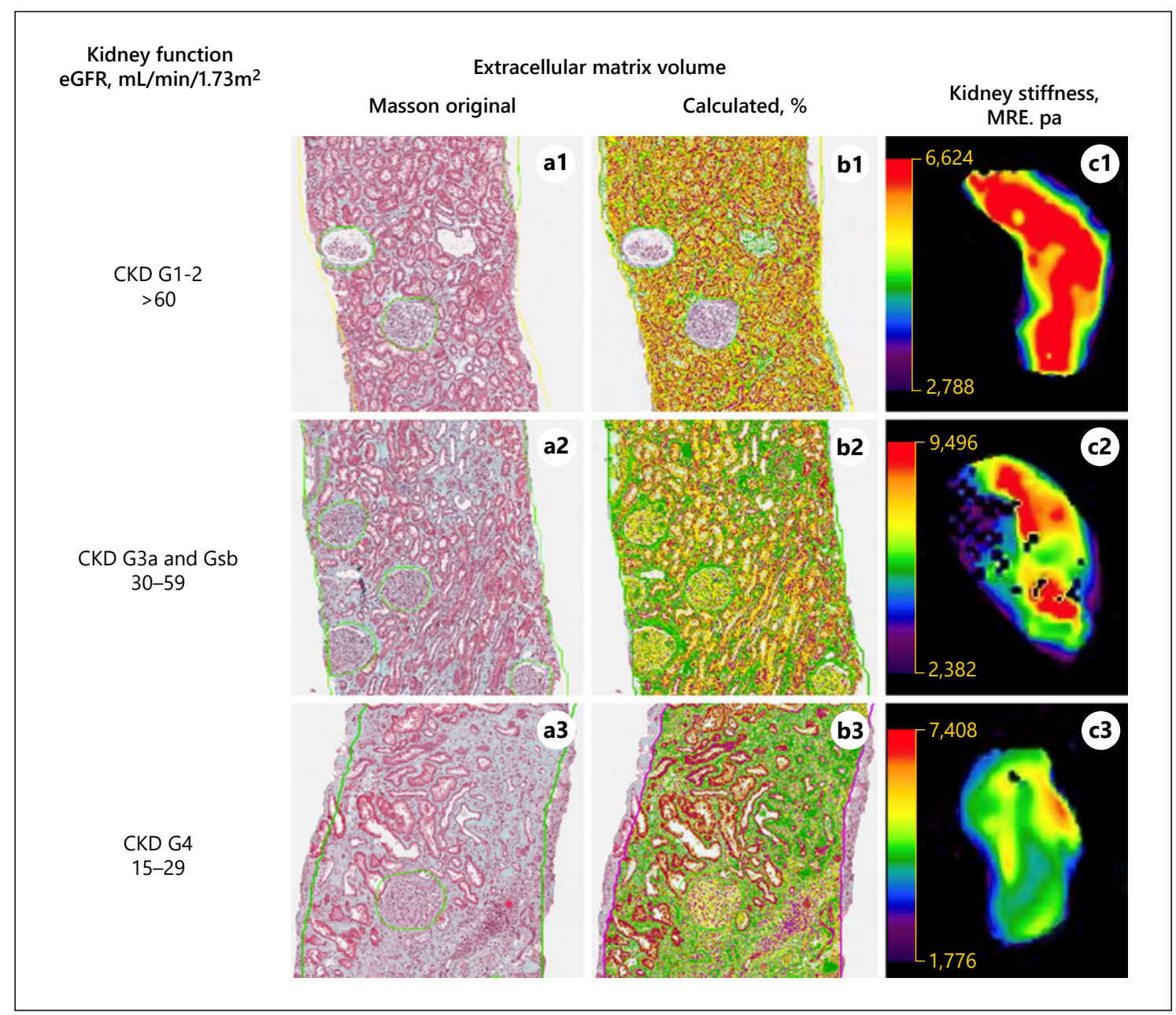

Fig. 1. Representative images of histology and MRE scanning showing kidney fibrosis. Representative images show histological fibrosis and kidney stiffness. As kidney function decreased from CKD G1 to G4, the Masson's slides showed increased extracellular matrix volume (a1-a3, b1-b3, original magnification, $\times 100)$. Kid- ney stiffness was generated from MRE scanning. The relevant stiffness colored images displayed as $\mathbf{c 1 - c 3}$ showed that the stiffness value decreased as kidney function decreased. MRE, magnetic resonance elastography.
Relationship between Cortex Interstitial Extracellular Matrix Volume, MRE, and eGFR

Representative images of histology and MRE scanning for kidney fibrosis are displayed in Figure 1. Cortex interstitial extracellular matrix volume data are summarized in online suppl. Table 2 . The cortex interstitial extracellular matrix volume increased in the later stages of $\mathrm{CKD}$, especially in CKD G3a, G3b, and G4. MRE measurements are summarized in online suppl. Table 3 . There was a significant difference in MRE between stages, and the values in CKD G3b and G4 were significantly lower than those in CKD G1.

First, we found a significant negative correlation between MRE-derived kidney stiffness and glomeruloscle- rosis ratio for these patients (Fig. 2a); Spearman's rho was $-0.460(p<0.001)$. As expected, we also found a significant negative correlation between MRE-derived kidney stiffness and cortex extracellular matrix volume (Fig. 2b); Spearman's rho was $-0.397(p<0.001)$. With increasing fibrosis, MRE values decreased in CKD kidneys.

The best mapping model of cortex interstitial extracellular matrix volume with MRE and eGFR was as follows:

\section{Model A:}

Interstitial Extracelluar Matrix Volume $=218.504-$ $14.651 \times \ln (\mathrm{MRE})-18.499 \times \ln (\mathrm{eGFR})$

The cortex interstitial extracellular matrix volume had a linear relationship with the natural logarithm of the 
Fig. 2. Relationship between cortex glomerulosclerosis ratio, cortex extracellular matrix volume, and MRE values in CKD. With increasing glomerulosclerosis ratios (a) and cortex extracellular matrix volumes (b), MRE values decreased in CKD kidneys. MRE, magnetic resonance elastography.

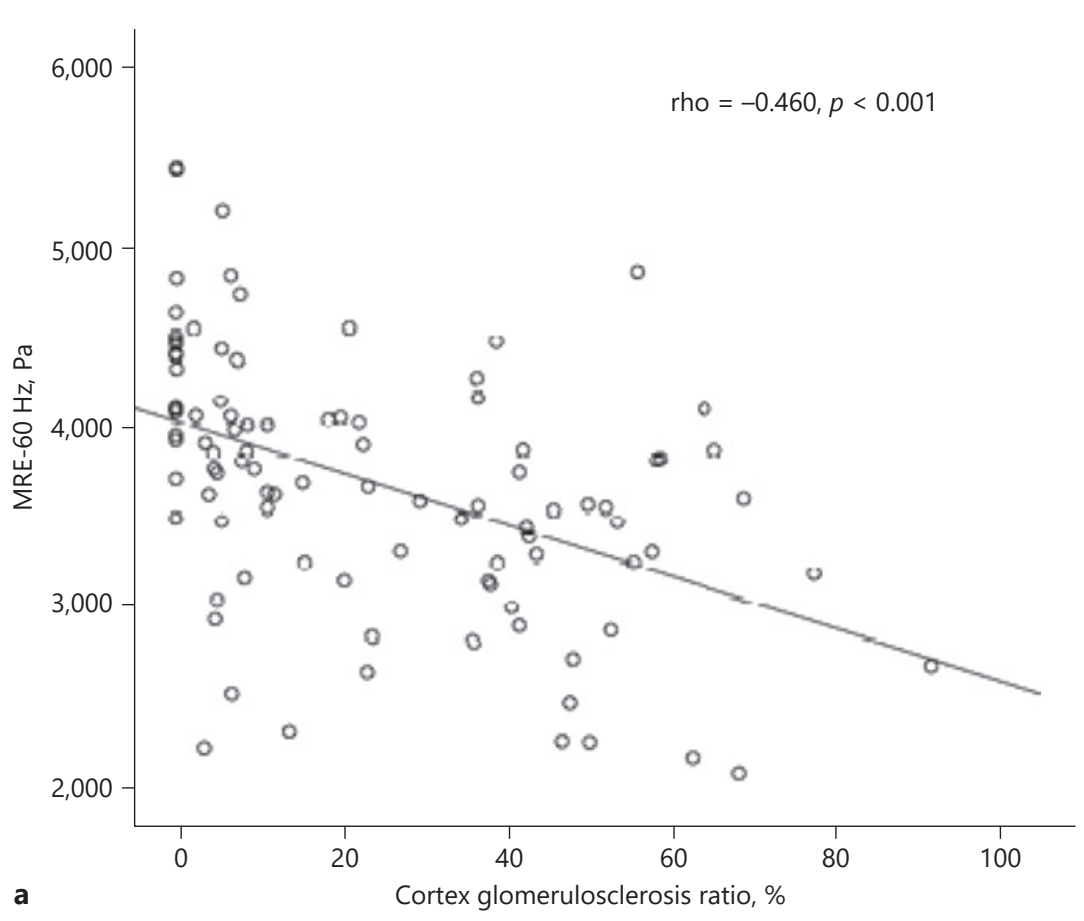

a

Cortex glomerulosclerosis ratio, \%

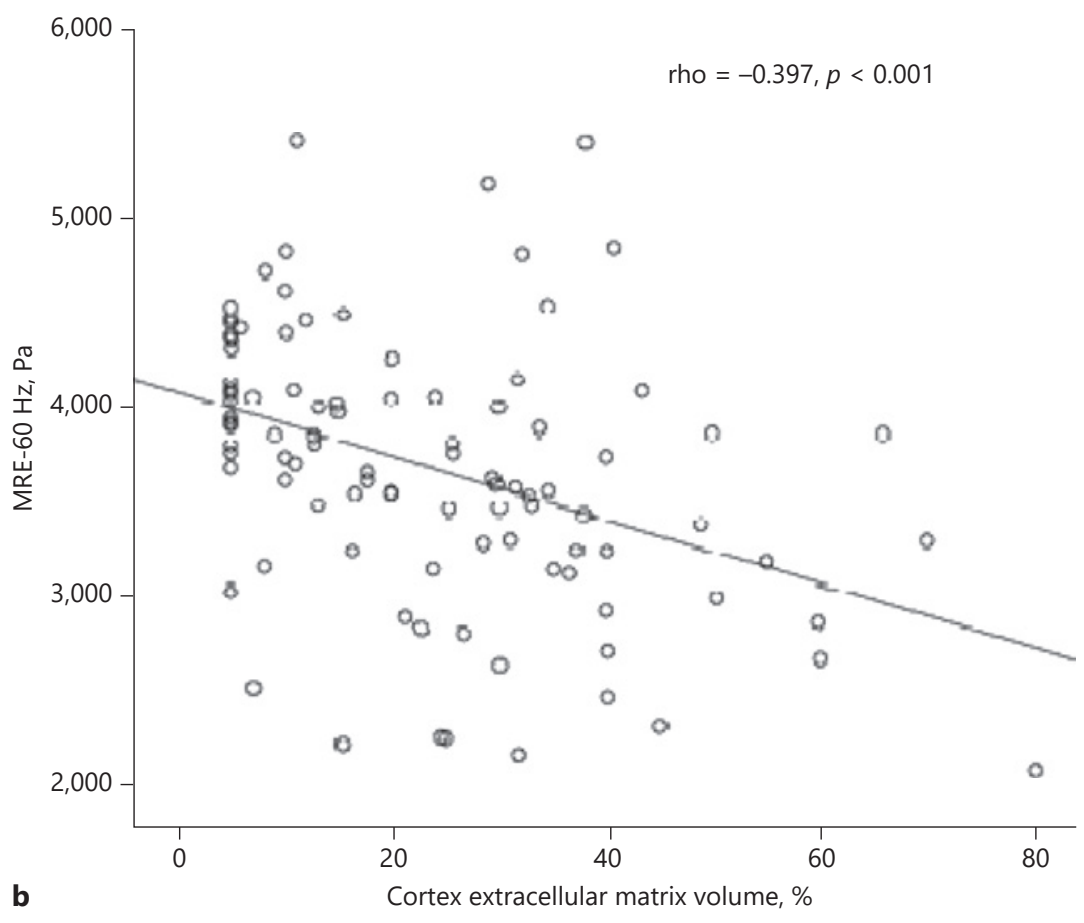

MRE value and eGFR. The $p$ values of each coefficient were $<0.05(6.16 \mathrm{e}-05,0.0326$, and $3.88 \mathrm{e}-10$ for the intercept, MRE value, and eGFR, respectively), implying a correlation between the 2 variables and extracellular matrix volume. As shown in Figure 3, it was obvious that the MRE value and eGFR were both negatively associated with the extracellular matrix volume. The lower the MRE value and eGFR are, the higher the cortex interstitial ex- 
Fig. 3. The best mapping model of kidney fibrosis with MRI and GFR. A model including cortex interstitial extracellular matrix volume, MRE, and eGFR was generated. From the $3 \mathrm{D}$ parametric graph, it was obvious that the MRE value and eGFR were both negatively associated with the extracellular matrix volume. The lower the MRE value and eGFR are, the higher the cortex interstitial extracellular matrix volume will be, which means more serious fibrosis in the kidney. Cortex interstitial extracellular matrix volume, \%; MRE, Pa; eGFR, mL/ min per $1.73 \mathrm{~m}^{2}$. MRI, magnetic resonance imaging; MRE, magnetic resonance elastography; eGFR, estimated glomerular filtration rate.

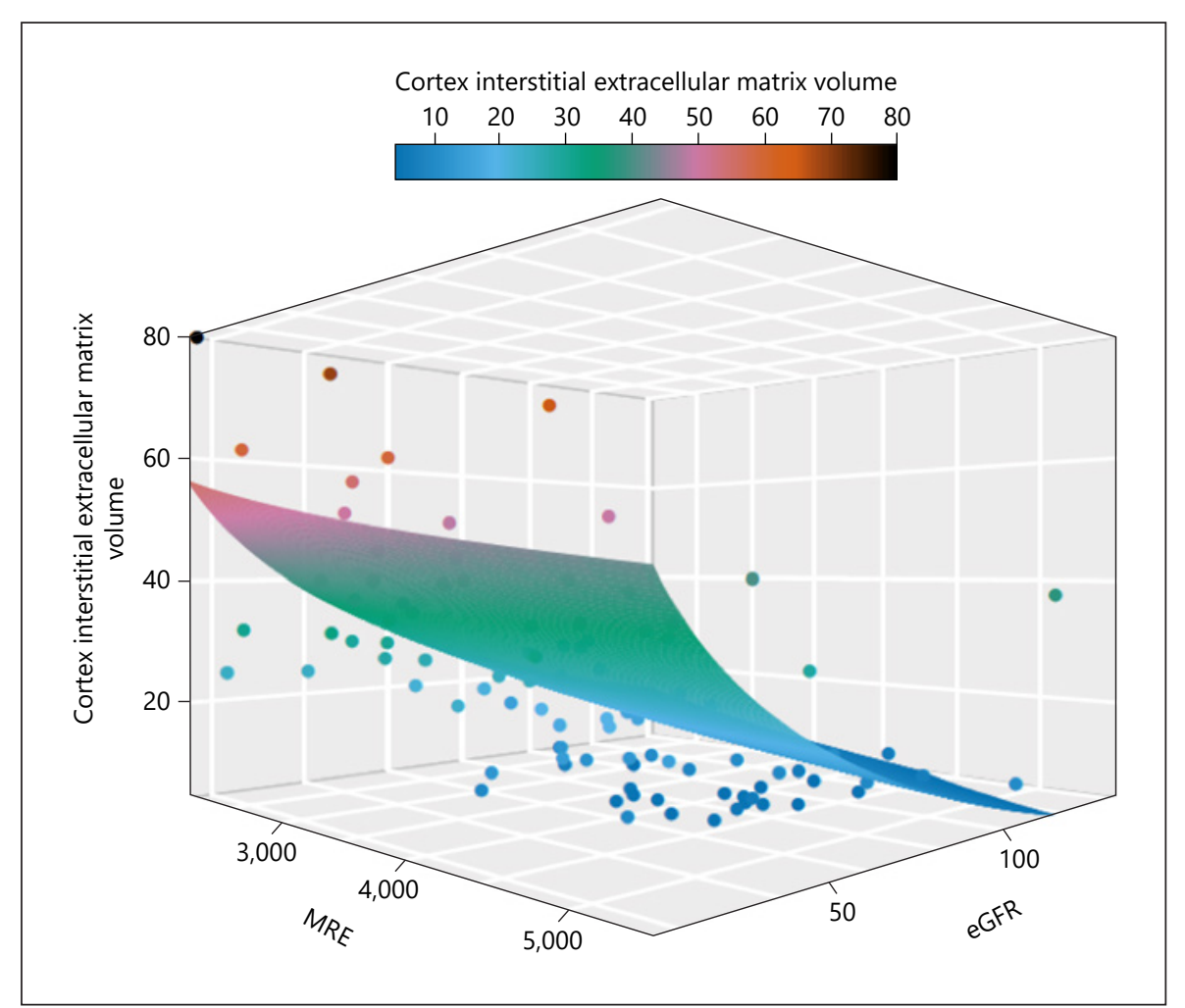

tracellular matrix volume will be, which means more serious fibrosis in the kidney. The evaluation statistics of model A are listed in online suppl. Table 4.

Next, we investigated whether MRE values could predict cortex fibrosis burden. We defined a $30 \%$ or over cortex interstitial extracellular matrix volume ratio as serious interstitial fibrosis and a $30 \%$ or over glomerulosclerosis ratio as serious glomerulosclerosis. For serious cortex interstitial fibrosis, the AUROC value was 0.667 (0.5550.766 ). The cutoff value was $3.5826 \mathrm{kPa}$, with $70 \%$ sensitivity and $69.1 \%$ specificity. For serious glomerulosclerosis, the AUROC value was 0.706 (0.595-0.801). The cutoff value was $3.5975 \mathrm{kPa}, 67.44 \%$ sensitivity, and $76.19 \%$ specificity.

\section{Relationship between Peritubular Capillary Density,} DWI-Fraction/DWI-Standard ADC, and eGFR

Representative images of immunohistochemistry and DWI scanning for kidney fibrosis are displayed in Figure 4. The cortex peritubular capillary densities were measured from CD34 staining and are shown in online suppl. Table 2. The peritubular capillary densities decreased in the later stages of CKD, especially in CKD G3a, G3b, and G4. The DWI-fraction and DWI-standard ADC mea- surements are summarized in online suppl. Table 3. There was a significant difference between stages in DWI-standard ADC, which had a significantly lower value in CKD G4 than in CKD G1. There was also a significant difference between stages in DWI-fraction, which was significantly lower in CKD G3b and G4 than in CKD G1.

We found that both the DWI-standard ADC and DWI-fraction values had significant positive correlations with peritubular capillary density (Fig. 5a, b); the Spearman's rho values were $0.244(p=0.016)$ and $0.472(p<$ $0.001)$, respectively, which indicated that DWI-fraction might be more sensitive than DWI-standard ADC for reflecting peritubular capillary density changes.

The best mapping model of peritubular capillary density with the DWI-standard ADC value or DWI-fraction value and eGFR was as follows:

Model B:

Peritubular Capillary Density $=17.914+9.403 \times$ $($ DWI - Fraction $)+0.112 \times($ eGFR $)$

Model C:

Peritubular Capillary Density $=22.136-0.809 \times$ $($ DWI - Standard ADC $)+0.130 \times($ eGFR $)$ 


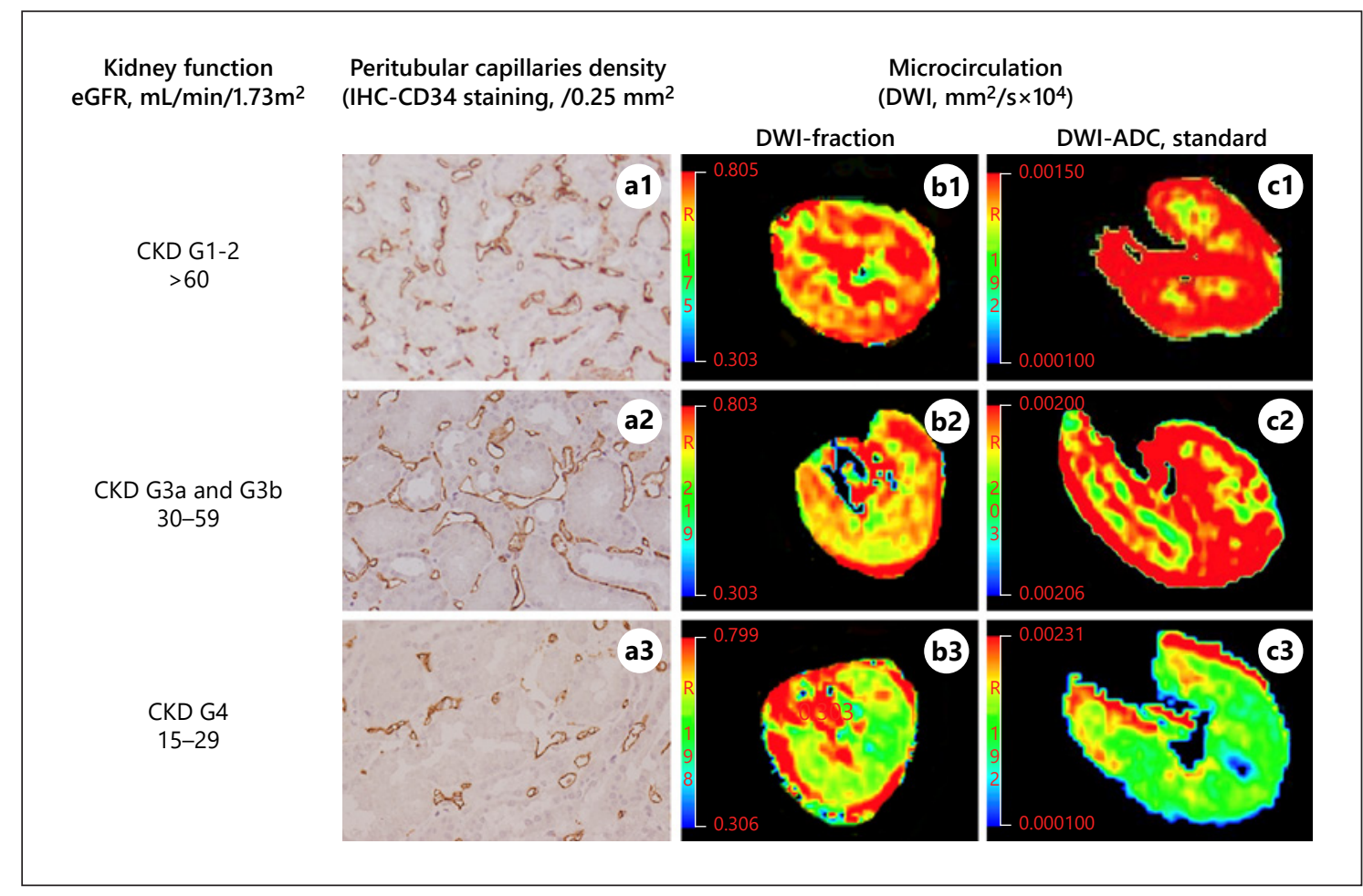

Fig. 4. Representative images of immunohistochemistry staining and DWI scanning showing kidney fibrosis. Representative images show peritubular capillaries and IVIM-DWI. As kidney function decreased, the CD34-positive capillary densities decreased (a1-a3, original magnification, $\times 400$ ). IVIM-DWI values were generated from DWI-frac- tion and DWI-standard ADC scanning, and the relevant colored images displayed as DWI-fraction (b1-b3) and DWI-standard ADC (c1-c3) showed that both of these tools decreased as kidney function decreased. IVIM-DWI, intravoxel incoherent motion diffusionweighted imaging; ADC, apparent diffusion coefficient.
The peritubular capillary density had a linear relationship with the DWI-fraction value and eGFR. For DWIfraction, the $p$ values of each coefficient were $<0.1$ $(2.3 \mathrm{e}-16,0.0584$, and $6.6 \mathrm{e}-10$ for the intercept, DWIfraction value, and eGFR, respectively). The relationship between DWI-fraction and peritubular capillary density was weaker with a relatively larger $p$ value. As shown in Figure 6a, DWI-fraction and eGFR were both positively associated with peritubular capillary density. The lower the eGFR and DWI-fraction, the lower the peritubular capillary density, which also indicates more serious kidney fibrosis.

For DWI-standard ADC, the $p$ values of each coefficient were $1.39 \mathrm{e}-07,0.728$, and $4.31 \mathrm{e}-13$ for the intercept, DWI-standard ADC, and eGFR, respectively. Figure $6 \mathrm{~b}$ shows that DWI-standard ADC was negatively associated with peritubular capillary density. However, this is irrelevant since we failed to reject the null hypothesis for DWI-standard ADC. The evaluation statistics of models $\mathrm{B}$ and $\mathrm{C}$ are listed in online suppl. Table 4.

Mapping Histology and MRI for Kidney Fibrosis

\section{Discussion}

There has been increasing interest in noninvasive imaging applications to CKD in the last 2 decades as evidencedbyinternational initiativessuch asthePARENCHIMA study that has published recently position papers and technical recommendations [28, 29]. Much of the reports to date have only related the imaging markers against renal functional markers such as eGFR. However, in the clinic, the limitations of eGFR especially at advanced stages are well recognized, and biopsy remains the confirmatory evidence. There are limited numbers of studies to date that have correlated imaging findings against biopsy. How can we help to fill in the gap between histology and imaging? We performed the current study that mapped histology features into MRI parameters by extensively reevaluating functional advanced imaging combined with kidney histology analysis.

Data to date using eGFR do show the differences in several MRI-derived parameters with disease severity in 
Fig. 5. Relationship between DWI-standard ADC, DWI-fraction, and peritubular capillaries in CKD. With decreasing peritubular capillary densities, DWI-standard $\operatorname{ADC}(\mathbf{a})$ and DWI-fraction (b) decreased in $\mathrm{CKD}$ kidneys. ADC, apparent diffusion coefficient.

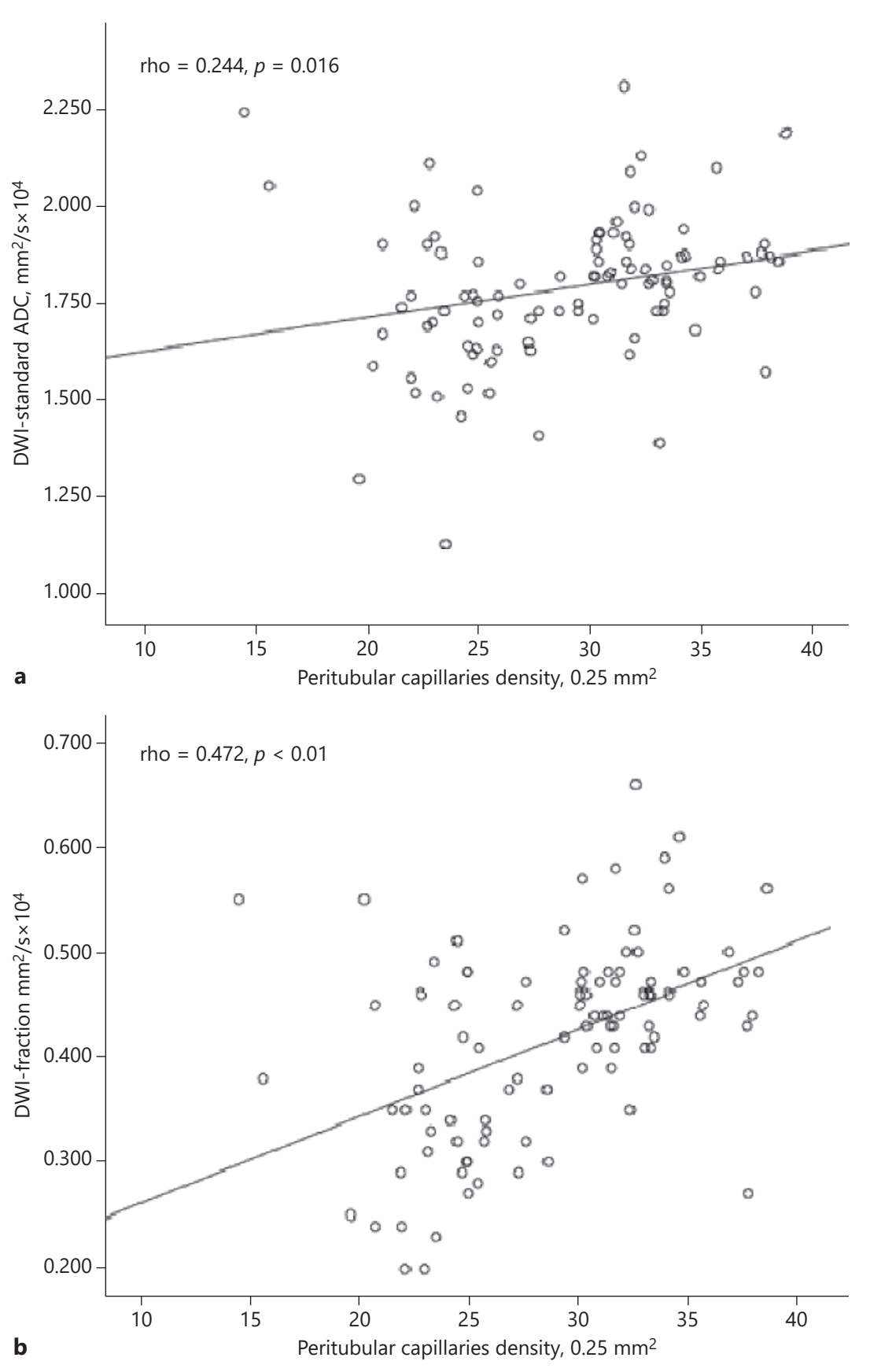

CKD. However, specificity of the dependence of each modality cannot be evaluated with correlation against eGFR. Biopsy remains the primary evidence for renal fibrosis. So, in the current work, we picked the interstitial extracellular matrix volume, which was evaluated by ImageScope (Aperio) from Masson's trichrome staining, and the peritubular capillary densities, which were quantitated by
CD34 staining from biopsy slices. Multiparametric quantitative MRI included MRE and IVIM-DWI. Our study first provides histological evidence to support that MRE and IVIM-DWI can effectively image fibrosis from the whole kidney, which supports a mapping relationship from imaging to histology. 
Fig. 6. The best mapping model of peritubular capillary densities with DWI-fraction/standard ADC and eGFR. a A model including peritubular capillary density, DWI-fraction, and eGFR was generated. From the 3D parametric graph, it was obvious that the DWI-fraction value and eGFR were both positively associated with the peritubular capillary density. The lower the DWI-fraction value and eGFR, the lower the density of peritubular capillaries, which means more serious fibrosis in the kidney. b The other model included peritubular capillary density, DWI-standard ADC, and eGFR. From the 3D parametric graph, it was obvious that the DWI-standard ADC value and eGFR were both positively associated with the peritubular capillary density, while the DWI-standard ADC value was weaker than eGFR. The lower the DWI-standard ADC value and eGFR, the lower the density of peritubular capillaries, which means more serious fibrosis in the kidney. Densities of peritubular capillaries, $10.25 \mathrm{~mm}^{2}$; DWI-fraction, $\mathrm{mm}^{2} / \mathrm{s} \times 10^{4}$; DWI-standard ADC, $\mathrm{mm}^{2} / \mathrm{s} \times 10^{4}$; eGFR, $\mathrm{mL} / \mathrm{min}$ per $1.73 \mathrm{~m}^{2}$. eGFR, estimated glomerular filtration rate; ADC, apparent diffusion coefficient.

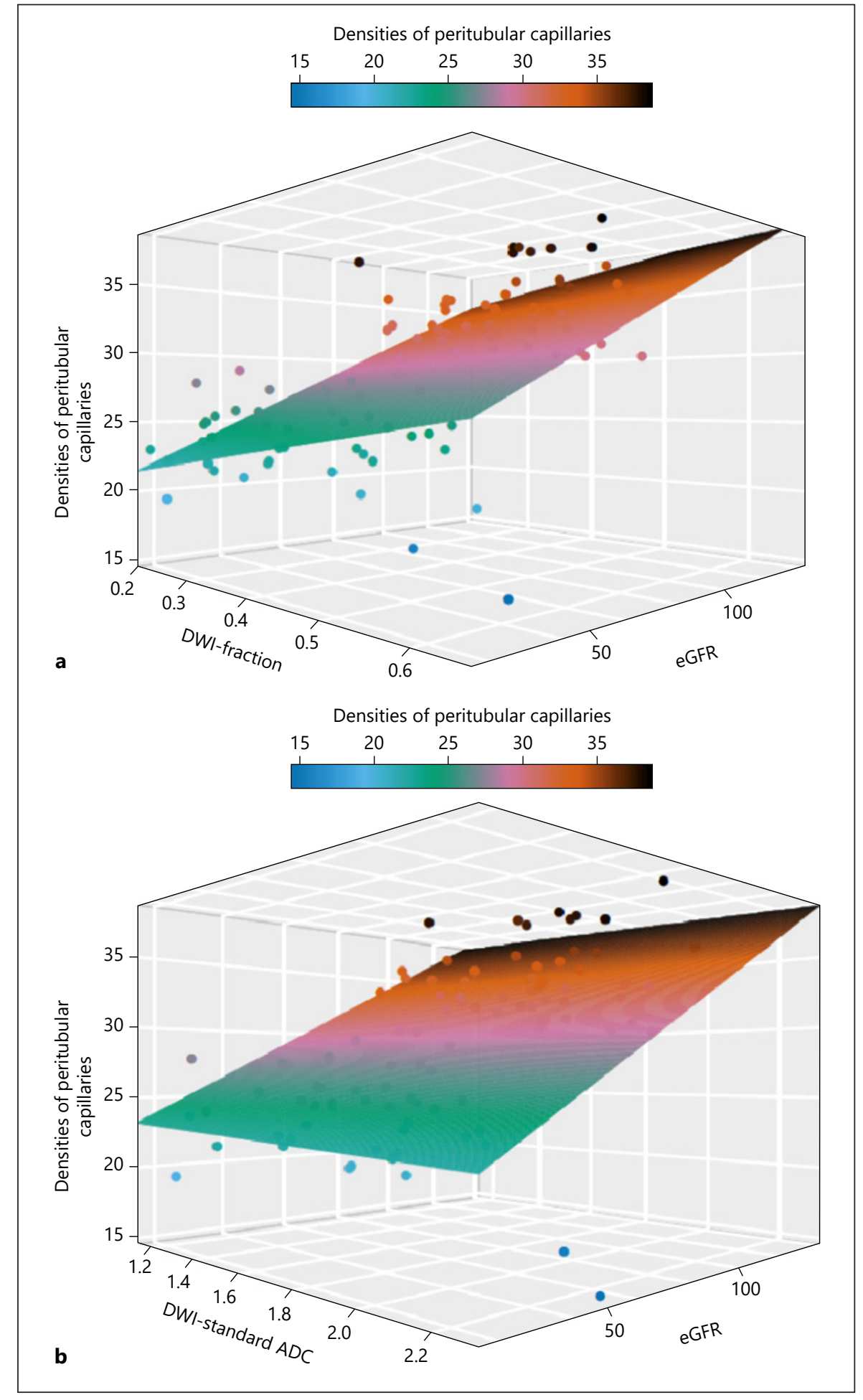

MRE combines imaging with the assessment of acoustic waves for the quantitative determination of the viscoelastic properties of tissues based on their response to external mechanical vibrations [29]. To date, MRE is a well- established technique for liver fibrosis imaging [30-32]; however, there are only a few high-quality studies evaluating MRE in human kidney allografts $[8,10,33]$ and in native kidneys $[9,15-17]$. The kidney possesses specific 
features rendering it more difficult to image since it is a heterogeneous organ. In addition, native kidneys are located quite deep and move with respiration, limiting the image quality and subsequent analysis. Moreover, the specificity of these techniques has been debated because they indirectly assess renal fibrosis by evaluating the GFR or histology. Most of the data from transplanted kidneys showed that MRE values were negatively associated with eGFR and fibrosis burden. Nevertheless, as authors from recent studies suggested, to our surprise, differing from that in the liver and allografts, MRE shear stiffness decreases with worsening CKD in IgA nephropathy [15], lupus nephritis [16], and diabetic nephropathy [17]. In IgA nephropathy, which is the most commonly studied glomerular nephritis, although 16 patients with biopsyproven IgAN were studied, the investigators found that advanced stage IgAN was associated with renal softening and restricted water diffusion [15]. A similar conclusion was also shown in a lupus nephritis study, which was still short of validation from histology features for MRI measurements [16].

Interestingly, we were able to find a negative correlation between MRE measurements and biopsy-derived cortex interstitial extracellular matrix volume from our data. The MRE data decreased with worsening CKD from the early stage to the late stage. We believe that our data clearly showed the mapping association between the cortex interstitial extracellular matrix and MRE imaging. We also showed a 3D parametric picture of the cortex extracellular matrix volume with MRE value and eGFR and were the first to combine these 3 parameters into 1 graph, which suggests that the interstitial extracellular matrix volume can be calculated by imaging and renal function. This equation might aid physicians in judging the kidney fibrosis burden from MRE and eGFR noninvasively.

DWI provides the quantification of the Brownian motion of water molecules by yielding the ADC. IVIM-DWI based on a biexponential fit model that separately evaluates tissue microperfusion and diffusion information may more exactly reveal the interaction of water molecule motion with tissue structures, which provides complete information about diffusion and perfusion properties $[6$, 7]. In 2011, Inoue et al. [5] briefly reported the noninvasive evaluation of kidney fibrosis and eGFR by using DWI-MRI in CKD and acute kidney injury patients. Mao et al. [34, 35] performed IVIM-MRI in CKD patients to assess functional and pathological alterations. Feng et al. [36] and Deng et al. [37] utilized IVIM-DWI to evaluate early GFR changes in type 2 diabetic patients and found that the IVIM parametric values were more sensitive than
ACR in detecting early-stage kidney changes. Recently, Bane et al. [38] performed multiparametric MRI and showed promising results for assessing renal transplant dysfunction with fibrosis, which included 15 biopsies confirming fibrosis. Friedli et al. [39] reported reduced ADC in multiparametric DWI-MRI to be the best predictor of "high" interstitial fibrosis, defined as $>40 \%$. Recently, Buchanan et al. [40] showed correlations between multiparametric MRI cortical ADC and eGFR $(r=0.54)$, and ADC values differed between low/high interstitial fibrosis groups at a $30-40 \%$ fibrosis threshold. This study showed that the multiparametric MRI measures, which were T1 mapping, DWI, arterial spin labeling perfusion, renal artery blood flow, and blood-oxygen-level-dependent MRI, have good reproducibility in $\mathrm{CKD}$, can distinguish $\mathrm{CKD}$ from healthy kidneys, and correlate with eGFR and interstitial fibrosis. Although promising, the diffusion MRI of abdominal organs is still difficult to use clinically because it is hard for the values to reflect the microcirculation pathophysiological situations in organs.

Capillary dropout and reduced microvascular blood flow are the key pathological characteristics of the fibrotic kidney [18]. Our data showed that both DWI-fraction and DWI-standard ADC had positive correlations with the peritubular capillary density. To our knowledge, this is the first report of the relationship between MRI and peritubular capillaries in native kidneys. These data showed the mapping association between cortex peritubular capillary densities and IVIM-DWI values. Some of these methods from this study have also been verified in transplanted kidneys from our center [23]. As Carloli et al. [41] reviewed, in few human studies where histology was available, cortical DWI-ADC displayed a good correlation with cortical fibrosis and chronic lesions. In our current study, the finding suggested that the peritubular capillary density can be estimated by the combination of IVIM-DWI and eGFR, as shown in the 3D parametric picture.

This study has limitations and the absence of followup data. There has been no previous investigation of the extracellular matrix, peritubular capillaries, and MRI in cortex kidneys. We realize that the performance of the derived models based on the current complete dataset in terms of generalizability is insufficiently clear for clinical practice. It would be better to have a holdout dataset to test the accuracy of the models developed on a subset of the data. We believe that in the way it has been applied and proposed, functional MRI would be very useful as a prognostic tool considering its ability to provide information about the degree of organ fibrosis [42-44]. 
In summary, our study provides histological evidence to support that MRI can effectively evaluate fibrosis in the kidney. These findings picture the graphs of the mapping model from imaging and eGFR into fibrosis, which has significant value for clinical implementation.

\section{Acknowledgements}

The authors are thankful to all the patients, nurses, and physicians who took part in this study. We also thank Dr. Yanjun Li and Dr. Shumin Tao for the technical assistance. Renal tissues were from the Renal Biobank of National Clinical Research Center of Kidney Diseases, Jiangsu Biobank of Clinical Resources.

\section{Statement of Ethics}

This research project was reviewed and approved by the Institutional Review Board of Jinling Hospital, Nanjing University School of Medicine, and conducted according to the principles of the Declaration of Helsinki (2017NZKY-013-01 and 2018NZGKJ-031). Informed written consent has been obtained from all participants.

\section{Conflict of Interest Statement}

The authors have no conflicts of interest to disclose.

\section{Funding Sources}

Jiong Zhang was supported by the grant National Key R\&D Program of China (2018YFC1312705), and Zhi-Hong Liu was supported by the grant National Key R\&D Program of China (precision medicine program, 2016YFC0904103).

\section{Author Contributions}

All authors contributed to the idea, conceptual advice, and framework of this manuscript. J.Z., Y.Y., and X.T. developed the research area and study design. J.Z. wrote the manuscript. X.L., G.X., and X.L. acquired the data and performed the statistical analysis. F.X. and M.Z. performed the histology work. L.Z. supervised the imaging work. Z.H.L. supervised and mentored the team.

\section{References}

1 Coresh J, Selvin E, Stevens LA, Manzi J, Kusek JW, Eggers P, et al. Prevalence of chronic kidney disease in the United States. JAMA. 2007; 298(17):2038-47.

2 Liu ZH. Nephrology in China. Nat Rev Nephrol. 2013;9(9):523-8.

3 Hewitson TD. Renal tubulointerstitial fibrosis: common but never simple. Am J Physiol Renal Physiol. 2009;296(6):F1239-44.

4 Klinkhammer BM, Goldschmeding R, Floege J, Boor P. Treatment of renal fibrosis-turning challenges into opportunities. Adv Chronic Kidney Dis. 2017;24(2):117-29.

5 Inoue $\mathrm{T}$, Kozawa E, Okada $\mathrm{H}$, Inukai $\mathrm{K}$, Watanabe S, Kikuta T, et al. Noninvasive evaluation of kidney hypoxia and fibrosis using magnetic resonance imaging. J Am Soc Nephrol. 2011 Aug;22(8):1429-34.

6 Thoeny HC, De Keyzer F. Diffusion-weighted MR imaging of native and transplanted kidneys. Radiology. 2011;259(1):25-38.

7 Eisenberger U, Binser T, Thoeny HC, Boesch C, Frey FJ, Vermathen P. Living renal allograft transplantation: diffusion-weighted MR imaging in longitudinal follow-up of the donated and the remaining kidney. Radiology. 2014;270(3):800-8.

8 Kirpalani A, Hashim E, Leung G, Kim JK, Krizova A, Jothy S, et al. Magnetic resonance elastography to assess fibrosis in kidney allografts. Clin J Am Soc Nephrol. 2017 Oct; 12(10):1671-9.

9 Rouvière O, Souchon R, Pagnoux G, Ménager JM, Chapelon JY. Magnetic resonance elas- tography of the kidneys: feasibility and reproducibility in young healthy adults. J Magn Reson Imaging. 2011 Oct;34(4):880-6.

10 Lee CU, Glockner JF, Glaser KJ, Yin M, Chen J, Kawashima A, et al. MR elastography in renal transplant patients and correlation with renal allograft biopsy: a feasibility study. Acad Radiol. 2012 Jul;19(7):834-41.

11 Korsmo MJ, Ebrahimi B, Eirin A, Woollard JR, Krier JD, Crane JA, et al. Magnetic resonance elastography noninvasively detects in vivo renal medullary fibrosis secondary to swine renal artery stenosis. Invest Radiol. 2013 Feb;48(2):61-8.

12 Litwiller DV, Mariappan YK, Ehman RL. Magnetic resonance elastography. Curr Med Imaging Rev. 2012;8(1):46-55.

13 Toguchi M, Tsurusaki M, Yada N, Sofue K, Hyodo T, Onoda M, et al. Magnetic resonance elastography in the assessment of hepatic fibrosis: a study comparing transient elastography and histological data in the same patients. Abdom Radiol. 2017 Jun; 42(6):1659-66.

14 Yin M, Talwalkar JA, Glaser KJ, Manduca A, Grimm RC, Rossman PJ, et al. Assessment of hepatic fibrosis with magnetic resonance elastography. Clin Gastroenterol Hepatol. 2007 Oct;5(10):1207-e2.

15 Lang ST, Guo J, Bruns A, Dürr M, Braun J, Hamm B, et al. Multiparametric quantitative MRI for the detection of IgA nephropathy using tomoelastography, DWI, and BOLD imaging. Invest Radiol. 2019 Oct;54(10):669-74.
16 Marticorena Garcia SR, Grossmann M, Bruns A, Dürr M, Tzschätzsch $\mathrm{H}$, Hamm B, et al. Tomoelastography paired with $\mathrm{T} 2 *$ magnetic resonance imaging detects lupus nephritis with normal renal function. Invest Radiol. 2019 Feb;54(2):89-97.

17 Brown RS, Sun MRM, Stillman IE, Russell TL, Rosas SE, Wei JL. The utility of magnetic resonance imaging for noninvasive evaluation of diabetic nephropathy. Nephrol Dial Transplant. 2020 Jun;35(6):970-8.

18 Leung G, Kirpalani A, Szeto SG, Deeb M, Foltz W, Simmons CA, et al. Could MRI be used to image kidney fibrosis? A review of recent advances and remaining barriers. Clin J Am Soc Nephrol. 2017 Jun;12(6): 1019-28.

19 Zhao J, Wang ZJ, Liu M, Zhu J, Zhang X, Zhang T, et al. Assessment of renal fibrosis in chronic kidney disease using diffusionweighted MRI. Clin Radiol. 2014 Nov;69(11): 1117-22.

20 Boor P, Perkuhn M, Weibrecht M, Zok S, Martin IV, Gieseke J, et al. Diffusion-weighted MRI does not reflect kidney fibrosis in a rat model of fibrosis. J Magn Reson Imaging. 2015 Oct;42(4):990-8.

21 Morrell GR, Zhang JL, Lee VS. Magnetic resonance imaging of the fibrotic kidney. J Am Soc Nephrol. 2017;28(9):2564-70.

22 Levey AS, Stevens LA, Schmid CH, Zhang YL, Castro AF 3rd, Feldman HI, et al. A new equation to estimate glomerular filtration rate. Ann Intern Med. 2009 May;150(9):604-12. 
23 Wang W, Yu Y, Wen J, Zhang M, Chen J, Cheng D, et al. Combination of functional magnetic resonance imaging and histopathologic analysis to evaluate interstitial fibrosis in kidney allografts. Clin J Am Soc Nephrol. 2019 Sep;14(9):1372-80.

24 Chen T, Li X, Li Y, Xia E, Qin Y, Liang S, et al. Prediction and risk stratification of kidney outcomes in IgA nephropathy. Am J Kidney Dis. 2019 Sep;74(3):300-9.

25 Wang Y, Zheng C, Xu F, Liu Z. Urinary fibrinogen and renal tubulointerstitial fibrinogen deposition: discriminating between primary FSGS and minimal change disease. Biochem Biophys Res Commun. 2016 Sep; 478(3):1147-52.

26 Ishii Y, Sawada T, Kubota K, Fuchinoue S, Teraoka $\mathrm{S}$, Shimizu A. Injury and progressive loss of peritubular capillaries in the development of chronic allograft nephropathy. Kidney Int. 2005 Jan;67(1):321-32.

27 Sun Q, Zhang M, Xie K, Li X, Zeng C, Zhou $\mathrm{M}$, et al. Endothelial injury in transplant glomerulopathy is correlated with transcription factor T-bet expression. Kidney Int. 2012 Aug;82(3):321-9.

28 Selby NM, Blankestijn PJ, Boor P, Combe C, Eckardt KU, Eikefjord E, et al. Magnetic resonance imaging biomarkers for chronic kidney disease: a position paper from the European Cooperation in Science and Technology Action PARENCHIMA. Nephrol Dial Transplant. 2018 Sep;33(Suppl 2):ii4-ii14.

29 Mendichovszky I, Pullens P, Dekkers I, Nery F, Bane O, Pohlmann A, et al. Technical recommendations for clinical translation of renal MRI: a consensus project of the Cooperation in Science and Technology Action PARENCHIMA. MAGMA. 2020 Feb;33(1): $131-40$.
30 Kim D, Kim WR, Talwalkar JA, Kim HJ, Ehman RL. Advanced fibrosis in nonalcoholic fatty liver disease: noninvasive assessment with MR elastography. Radiology. 2013 Aug; 268(2):411-9.

31 Tan CH, Venkatesh SK. Magnetic resonance elastography and other magnetic resonance imaging techniques in chronic liver disease: current status and future directions. Gut Liver. 2016;10(5):672-86.

32 Manduca A, Oliphant TE, Dresner MA, Mahowald JL, Kruse SA, Amromin E, et al. Magnetic resonance elastography: non-invasive mapping of tissue elasticity. Med Image Anal. 2001 Dec;5(4):237-54.

33 Marticorena Garcia SR, Fischer T, Dürr M, Gültekin E, Braun J, Sack I, et al. Multifrequency magnetic resonance elastography for the assessment of renal allograft function. Invest Radiol. 2016 Sep;51(9):591-5.

34 Mao W, Zhou J, Zeng M, Ding Y, Qu L, Chen C, et al. Chronic kidney disease: pathological and functional evaluation with intravoxel incoherent motion diffusion-weighted imaging. J Magn Reson Imaging. 2018 May;47(5): 1251-9.

35 Mao W, Zhou J, Zeng M, Ding Y, Qu L, Chen $\mathrm{C}$, et al. Intravoxel incoherent motion diffusion-weighted imaging for the assessment of renal fibrosis of chronic kidney disease: a preliminary study. Magn Reson Imaging. 2018 Apr;47:118-24

36 Feng YZ, Chen XQ, Yu J, Liu XL, Cheng ZY, Ren WW, et al. Intravoxel incoherent motion (IVIM) at $3.0 \mathrm{~T}$ : evaluation of early renal function changes in type 2 diabetic patients. Abdom Radiol. 2018 Oct;43(10):2764-73.

37 Deng Y, Yang B, Peng Y, Liu Z, Luo J, Du G. Use of intravoxel incoherent motion diffusion-weighted imaging to detect early changes in diabetic kidneys. Abdom Radiol. 2018 Oct;43(10):2728-33.
38 Bane O, Hectors SJ, Gordic S, Kennedy P, Wagner M, Weiss A, et al. Multiparametric magnetic resonance imaging shows promising results to assess renal transplant dysfunction with fibrosis. Kidney Int. $2020 \mathrm{Feb}$;97(2): 414-20.

39 Friedli I, Crowe LA, Berchtold L, Moll S, Hadaya $K$, de Perrot $T$, et al. New magnetic resonance imaging index for renal fibrosis assessment: a comparison between diffusionweighted imaging and T1 mapping with histological validation. Sci Rep. 2016 Jul;6: 30088.

40 Buchanan CE, Mahmoud H, Cox EF, McCulloch T, Prestwich BL, Taal MW, et al. Quantitative assessment of renal structural and functional changes in chronic kidney disease using multi-parametric magnetic resonance imaging. Nephrol Dial Transplant. 2020 Jun;35(6): 955-64.

41 Caroli A, Schneider M, Friedli I, Ljimani A, De Seigneux S, Boor P, et al. Diffusionweighted magnetic resonance imaging to assess diffuse renal pathology: a systematic review and statement paper. Nephrol Dial Transplant. 2018 Sep;33(Suppl 2):ii29-40.

42 Poggio ED. Imaging as a noninvasive tool for evaluating interstitial fibrosis in kidney allografts. Clin J Am Soc Nephrol. 2019;14(9): $1286-7$

43 Zhang J, Zhang LJ. Functional MRI as a tool for evaluating interstitial fibrosis and prognosis in kidney disease. Kidney Dis. 2020 Jan; 6(1):7-12.

44 Hirakawa Y, Nangaku M. Visualizing fibrosis-hope for ideal markers beyond imaging. Kidney Int. 2020 Mar;97(3):437-8. 